\title{
Design of Zero Head Turbines for Power Generation
}

\author{
Edwin Chica and Ainhoa Rubio-Clemente
}

Additional information is available at the end of the chapter

http://dx.doi.org/10.5772/66907

\begin{abstract}
Failure analysis of the blades of a horizontal axis hydrokinetic turbine of $1 \mathrm{~kW}$ is presented. Analysis consisted of the determination of the pressure on the blade surface using Computational Fluid Dynamics, and the calculation of the stress distribution in the blade due to hydrodynamic, inertial and gravitational loads using the finite element methods. The results indicate that the blade undergoes significant vibration and deflection during the operation, and the centrifugal and hydrodynamic loads considerably affect the structural response of the blade; however, the stresses produced in all of the analysed models did not exceed the safe working stresses of the materials used to manufacture the blade. Modal analysis was conducted to calculate first significant natural frequencies. Results were studied in depth against operating frequency of the turbine. After carrying out the modal analysis, harmonic analysis was also done to see the response of the turbine under dynamic loading. It was observed that the turbine is safe in its entire operating range as far as phenomenon of resonance is concerned. Additionally, it was observed that maximum harmonic response of the turbine on the application of dynamic loading is far lesser than its failure limit within the specified operating range.
\end{abstract}

Keywords: horizontal axis hydrokinetic turbine, tip-speed ratio, power coefficient, blade design, blade load

\section{Introduction}

In order to generate new and sustainable solutions for the energy crisis and climate change, many alternatives have been studied for increasing the potential of use of renewable energy systems. The widely known alternative energy resources are solar, wind, wave, hydraulic, biomass, and geothermal, among others. However, hydrokinetic energy is one of the renewable energies gaining a great global attention in recent years, especially because it is a stable, continuous, sustainable, and high-energy density renewable resource. Moreover, its costs and 
development time are small, resulting in a minimal environmental impact. In addition, many countries have a large hydrokinetic potential thanks to their long rivers with stable and high flow rates $[1,2]$.

Hydrokinetic systems convert the energy of moving water in rivers, oceans, or tidal currents into electricity without the use of a dam or barrage associated to conventional hydropower. Nevertheless, hydrokinetic energy conversion systems are still in early stages of development; therefore, further researches are required [1,2]. The main challenges linked to the use of hydrokinetic energy are not only to have efficient systems but also to convert energy in a more economical way so that the cost-benefit analysis leads to the growth of this alternative energy generation. For the hydrokinetic energy extraction from water, a turbine submerged in the flowing stream of water is needed, which must be designed for operating conditions of the location where it will be placed to obtain the highest efficiency production. The turbine should be ideally installed at locations that have relatively steady flow throughout the year and are not prone to serious flood events, turbulence, or extended periods of low water level [2-4].

There are several kinds of hydrokinetic turbines of various sizes and different energy capture principles. The major classification of hydrokinetic turbines is related to the rotating axis positions with respect to the water flow: (a) horizontal axis hydrokinetic turbines (HAWTs), where the rotational axis must be oriented in parallel with regard to the water current in order to produce power and (b) vertical axis hydrokinetic turbines (VAWTs), where the rotational axis is perpendicular to the water current direction $[4,5]$.

Regardless the type of turbine, HAWTs and VAWTs are still at a pre-commercial stage, although a few of them have been developed at a full scale. This fact can be adequate to decentralize the electricity generation in off-grid remote communities, particularly, in developing countries where the kinetic energy conversion may be possible due to the strong currents present in the rivers. In this sense, these ones might be installed in isolated or grid-connected configuration, standing alone, or as a supplement to an existing generation system, floating or fixed to the bottom of the water current. Their construction methods and materials should be appropriate for their local manufacture in these countries [3, 5-7].

The design philosophies of hydrokinetic turbines are similar to that of wind turbines, sharing the same working principles, which consist of converting the hydrokinetic power into mechanical power in the form of rotating blades. In general, many hydrokinetic turbine researches are focused on accurately predicting their efficiency. Various computational models exist, each one with their own strengths and weaknesses, attempting to precisely predict the performance of a hydrokinetic turbine. Being able to numerically predict the hydrokinetic turbine performance offers the possibility to reduce the number of the required experimental tests. Additionally, computational studies are more economical than the costly experiments [5-7].

It is noteworthy that numerous sources claim that HAWTs have a major efficiency per same swept area. Nevertheless, the main advantage of VAWTs is that the blades can have a constant shape along their length and, unlike HAWTs, twisting the blade is not required, as every section of the blade is subjected to the same water speed, allowing an easier design, fabrication, and replication of the blade, which can influence in a cost reduction. This is the reason why this 
kind of rotor configuration for the design of hydrokinetic turbines is selected. Nonetheless, VAWTs are not as efficient as HAWTs, and they exhibit a very low starting torque, as well as dynamic stability problems [5-7].

The structural design of HAWT blades is also as important as their hydrodynamic design due to the dynamic structural loads a rotor may experience play the major role in determining its lifetime. For this purpose, modal analysis provides information on the dynamic characteristics of structural elements at resonance and, thus, it helps in understanding their detailed dynamic behavior. It is important to note that a good hydrokinetic system should be able to mitigate the unwanted vibratory response, which can only be done by avoiding resonance. Hence, modal or resonance frequency should be computed during the design phase of the blade $[6,7]$. Hydrodynamic loads are a major source of dynamic structural behavior and ought to be accurately determined. Under this scenario, this work is aimed at contributing to the better comprehension of the structural static and dynamic behavior of three internal blade configurations using Computational Fluid Dynamic (CFD) simulations and Finite Element Analysis (FEA).

\section{Hydrodynamic design of a horizontal axis hydrokinetic turbine}

The performance of a hydrokinetic turbine is a main concern in the design stage. It depends on the interaction between the rotor and the water current. The rotor is the most important component in a hydrokinetic turbine, which must be designed for capturing water energy and converting it into rotating mechanical energy. The rotor consists of several blades joined to a common hub $[6,7]$. The turbine blades are critical components of the rotor, which use the flow to cause a pressure difference on either side of the hydrofoil profile, creating a resultant force on the blade profile and, subsequently, causing a rotational movement. This rotating movement of the rotor shaft is passed, in most cases, through a gearbox to increase the amount of revolutions of the moving rotor. The output of the gearbox is directly coupled to a generator, which is used to produce electricity and transfer it to the desired location via cables $[1,4,5,8]$.

The geometry and dimension of the rotor are determined by the performance requirements of the hydrokinetic turbine. In general, two fundamental issues must be considered simultaneously in the rotor design process: the hydrodynamic performance and the structural design. The hydrodynamic characteristics of the turbine are influenced by the chosen hydrofoil. Generally, an optimum turbine blade is narrower at the tip and wider near the root. It must be designed to meet structural requirements and it can be manufactured easily. There are also other important design considerations such as the blade materials, noise reduction, condition/health monitoring, blade roots, and hub attachment, among others $[3,4]$.

The design methods of the main characteristics of the turbine rotor are based on the same incompressible flow techniques used for designing wind turbines [4, 5, 9]. The first step involved in the rotor design is to fix the turbine output power and estimate the blade radius. 
For this purpose, it is widely known that the energy in moving water is in the form of kinetic energy, which may be expressed using Eq. (1):

$$
E=1 / 2 m V_{1}^{2}
$$

where $m$ and $V_{1}$ correspond to the mass and the velocity of the object, respectively. In a hydrokinetic turbine, there are several units of water masses moving perpendicularly to the plane or area $(A)$ swept out by the blades. This area can be expressed as $A=\pi R^{2}$, where $R$ is the blade radius. In turn, the power $(P)$ is the rate of energy movement per unit of time or the rate at which energy is generated or consumed per unit time. Mathematically, $P$ is the first derivative of the energy taken with respect to time $(d E / d t)$. This power is developed in the hydrokinetic turbine from the mass movement of water, as indicated in Eq. (2):

$$
P=\frac{d E}{d t}=\frac{1}{2} \frac{\mathrm{dm}}{d t} V_{1}^{2}
$$

The next step is to quantify the amount of water flow associated with $d m / d t$. Given a unit of time $(t)$, the unit of water masses $(m)$ is moved a distance of $L$. This can be used to arrive at two important results. First, the first derivative of $L$ with respect to $t$ is $d L / d t$, which is equal to velocity $\left(V_{1}\right)$. Second, the volume of water passing through $A$ will be $A L$.

If the density of water $(\rho)$ is known or can be found, then the mass of water moving through the plane $A$ is $m=\rho A L$. The mass movement rate $d m / d t$ is equal to $\rho A d L / d t$ or the expression represented in Eq. (3):

$$
\frac{\mathrm{dm}}{d t}=\rho A V_{1}
$$

Substituting Eq. (3) into Eq. (2), Eq. (4) is obtained:

$$
P=\frac{1}{2} \rho A V_{1}^{3}
$$

Eq. (4) expresses the ideal power in a fluid flow. However, hydrokinetic turbines are limited by blade efficiencies, mechanical losses in transmissions, electrical losses, and the theoretical amount of energy allowed to be extracted from the water current. Because of these losses and inefficiencies, two more variables must be added to Eq. (4). The first variable is $\eta$, which is a measure of the efficiency of the gearbox, the electrical inverter, and the generator. This variable takes into account all the friction, slippage, and heat losses associated with the internal mechanical and electrical components. Values of $\eta$ may greatly differ among the different turbine models. A reasonable and conservative value of $\eta$ can be around $70 \%[1,8]$.

The second variable is the power coefficient $\left(C_{p}\right)$, which refers to a measure of the blade or hydrofoil efficiency. It includes the hydrofoil shape and the hydrodynamic forces of lift and drag. The $C_{p}$ expresses the hydrofoil ability to transform the water kinetic energy into mechanical power, which is delivered to the turbine transmission or directly to the electrical generator. A higher $C_{p}$ is preferred over a lower $C_{p}$ value. These $C_{p}$ values may vary with the turbine size. 
Placing these two variables, $\eta$ and $C_{p^{\prime}}$ into Eq. (4), the following expression is obtained:

$$
P=\frac{1}{2} \rho A V_{1}^{3} C_{p} \eta
$$

Eq. (5) is considered to determine the power of the hydrokinetic turbine. $P$ is the net power derived from water after accounting for losses and inefficiencies. From this equation, it can be seen that the extractable power is dependent on the turbine power coefficient, the density of the flow medium, the swept area of the turbine, and the mean velocity of water $\left(V_{1}\right)[8]$.

If the water density, blade radius, and water velocity are constant, the captured power $(P)$ is proportional to the $C_{p}$, which depends on the tip-speed ratio (TSR) $(\lambda)$ and the pitch angle of the turbine $(\theta)$, as in wind turbines. The maximization of $C_{p}$ is of fundamental importance in order to optimize the extraction of energy from water. It is highlighted that $\lambda$ is the ratio between the speed of the blade at its tip and the speed of the water current. This ratio has a strong influence on the efficiency of the turbine $[4,5,8,10]$. This variable may be defined as Eq. (6):

$$
\lambda=\frac{R \omega}{V_{1}}
$$

where $\omega$ is the rotational speed of the rotor of the hydrokinetic turbine (rad/s). The calculation of the $C_{p}$ requires the use of the blade element theory. However, numerical approximations have been developed [11]. In the current work, Eq. (7) was used:

$$
\begin{gathered}
C_{p}(\lambda, \theta)=0.22\left(\frac{116}{\lambda_{i}}-0.4 \theta-5\right) e^{\frac{-12.5}{\lambda_{i}}} \\
\frac{1}{\lambda_{i}}=\frac{1}{\lambda+0.08 \theta}-\frac{0.035}{\theta^{3}+1}
\end{gathered}
$$

With the function defined in Eq. (8), it is possible to evaluate $C_{p}$ at different values of tip-speed ratio and pitch angle. This leads to the $C_{p}(\lambda, \theta)$ versus $\lambda$ characteristics for various values of $\theta$ as depicted in Figure 1.

In turn, Figure 2 shows that for every $\theta$, there is a $\lambda$, which corresponds to the maximum $C_{p}$ and, hence, to the maximum efficiency. Note that in Figure 2, $C_{p}$ has a maximum at 0.4382 when $\theta$ and $\lambda$ are equal to 0 and 6.325, respectively. This has two important connotations. On the one hand, achieving a maximum at $\theta=0$ means that any $\theta$ deviation yields lower power capture. On the other hand, maximum conversion efficiency is accomplished at $\lambda=6.325$.

Before using Eq. (6), $V_{1}$ have to be determined or assumed. In this work, $V_{1}$ was assumed to be $1.5 \mathrm{~m} / \mathrm{s}$; the turbine output power, $1 \mathrm{~kW}$; the drive train efficiency, $70 \%$; and $\rho, 997 \mathrm{~kg} / \mathrm{m}^{3}$ $\left(25^{\circ} \mathrm{C}\right)$. Now, the radius and, subsequently, the length of the turbine blades may then be calculated. The required blade length resulted to be equal to $0.79 \mathrm{~m}$.

The analysis of the hydrodynamic behavior of hydrokinetic turbine can be done similarly to the simplest model of a wind turbine. Therefore, a simple model, generally attributed to Betz 


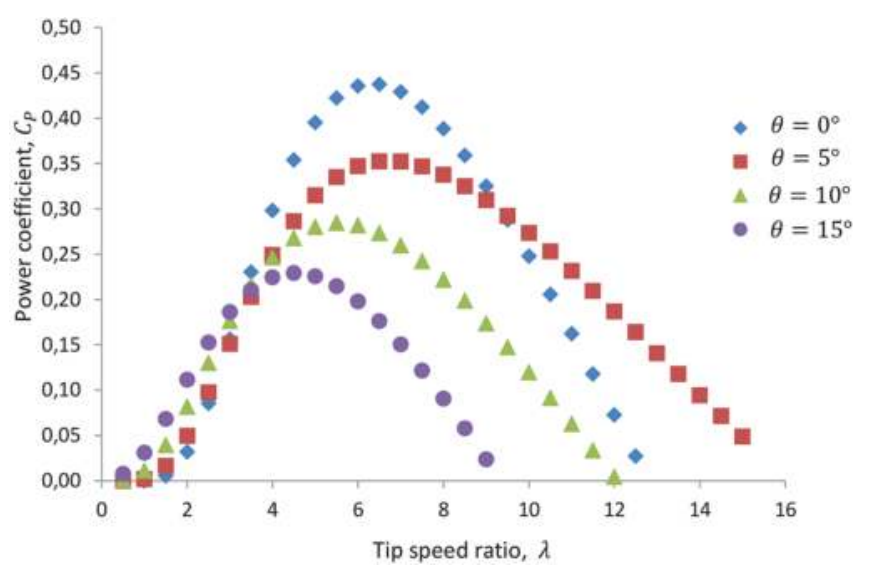

Figure 1. Power coefficient $\left(C_{p}\right)$ as a function of the tip-speed ratio $(\lambda)$ with the pitch angle $(\theta)$ as a parameter.

(1926), can be used to determine the power, the thrust of the water on the ideal rotor, and the effect of the rotor operation on the local water field $[4,5,9,12]$. The referred model is known as "actuator disk model" where the rotor is replaced by a homogenous disk extracting energy from water. Actuator disk model is based on the assumptions like no frictional drag, homogenous, incompressible, steady-state fluid flow, constant pressure increment or thrust per unit area over the disk, continuity through the disk, and an infinite number of blades. The analysis of the actuator disk model assumes a control volume as illustrated in Figure 2.

The analysis of the actuator disk theory assumes a control volume in which the boundaries are the surface walls of a stream tube and two cross sections. In order to analyze this control volume, four stations (1: free stream region, 2: just before the blades, 3: just after the blades, 4: far wake region) need to be considered (Figure 2). Applying the conservation of linear momentum equation on both sides of the actuator disk, the net force on the contents of the control volume can be found. That force is equal and opposite to the thrust $(I)$, which is the force of water on the hydrokinetic turbine. $I$ is equal and opposite to the change in momentum of water stream. For steady-state flow, assuming the continuity of mass flow, I can be obtained as expressed in Eq. (9):

$$
I=m\left(V_{1}-V_{4}\right)=m_{2}\left(V_{1}-V_{4}\right)=\rho A_{2} V_{2}\left(V_{1}-V_{4}\right)
$$

I can be also expressed as the sum of the force on each side of the actuator disk, as represented in Eq. (10):

$$
I=A_{2}\left(P_{2}-P_{3}\right)
$$

Since $I$ is positive, the velocity behind the rotor $\left(V_{4}\right)$ is less than the free stream velocity $\left(V_{1}\right)$. On the other hand, as the flow is frictionless and no work or energy transfer is done, Bernoulli 

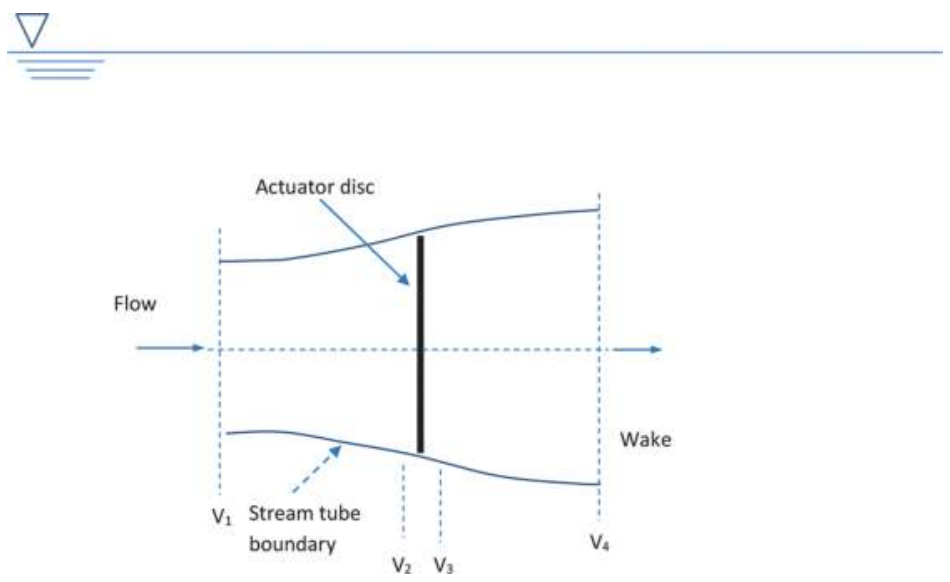

Figure 2. Idealized flow through a hydrokinetic turbine represented by a nonrotating actuator disk.

equation can be applied on both sides of the rotor. If energy conservation using Bernoulli equation is applied between stations 1 and 2 and stations 3 and 4, and they are combined, it is possible to find the pressure decrease $\left(P_{2}-P_{3}\right)$ :

$$
\left(P_{2}-P_{3}\right)=\frac{1}{2} \rho\left(V_{1}^{2}-V_{4}^{2}\right)
$$

Equating I values from Eqs. (9) and (10) and substituting Eq. (11), Eq. (12) can be obtained:

$$
V_{2}=\frac{V_{1}+V_{4}}{2}
$$

The water velocity at the rotor plane, using the simple model, is the average of the upstream and downstream water speeds. If the axial induction factor $(a)$ is defined as the fractional decrease in water velocity between the free stream and the rotor plane, then Eq. (13) can be achieved:

$$
a=\frac{V_{1}-V_{2}}{V_{1}}
$$

Substituting Eq. (13) into Eq. (12), Eqs. (14) and (15) can be obtained:

$$
\begin{gathered}
V_{2}=V_{1}(1-a) \\
V_{4}=V_{1}(1-2 a)
\end{gathered}
$$

The power captured by a hydrokinetic turbine rotor $(P)$ is equal to $I$ times the velocity at the rotor plane: 


$$
P=\rho A_{2} V_{2}\left(V_{1}-V_{4}\right) V_{2}
$$

Substituting Eqs. (14) and (15) into Eq. (16), Eq. (17) can be achieved:

$$
P=2 \rho A_{2} V_{1}^{3} a(1-a)^{2}
$$

Equating the power values from Eqs. (17) and (5), Eq. (18) is attained:

$$
C_{p}=\frac{4 a(1-a)^{2}}{\eta}
$$

The maximum $C_{p}$ is determined by taking the derivative of Eq. (18) with respect to $a$ and setting it equal to zero, which yields Eq. (19) when $a=1 / 3$ [9]:

$$
C_{p}=\frac{16}{27}=0.5926
$$

This result indicates that if an ideal rotor is designed and operated so that the water speed at the rotor is $2 / 3$ of the free stream water speed, then it would be operating at the point of maximum power production. This is known as the Betz limit [9]. Thereby, for the hydrokinetic turbine of the best rotor design, extracting more than about $60 \%$ of the kinetic water energy is evidently non possible. In the design of the horizontal axis hydrokinetic turbine of $1 \mathrm{~kW}$, the value of $C_{p}=0.5926$ was not used. The used value was obtained from Eq. (7) [9]. However, the value of $a$ was determined from Eq. (18) for a $C_{p}$ equal to 0.4382 and $\eta$ equal to $100 \%$. In this case, $a$ was 0.15254 .

In order to make the results more realistic, the effect of wake rotation should be considered. Two assumptions were made to describe this effect. The upstream flow was assumed to be entirely axial, while the downstream flow was assumed to have rotation at an angular speed $(\Omega)$. By considering the tangential flow behind the rotor, the angular induction factor $\left(a^{\prime}\right)$ was introduced [9]:

$$
a^{\prime}=\frac{\Omega}{2 \omega}
$$

where $\Omega$ and $\omega$ refer to the induced tangential angular velocity of the flow and the angular velocity of the rotor, respectively. Equations for the torque and the thrust force can be obtained by considering the flow through an annular cross section of radius $r$ with area $2 \pi r d r$. Thus, the experienced thrust force and torque can be expressed as Eqs. (21) and (22), respectively:

$$
\begin{gathered}
d I=4 a^{\prime}\left(1+a^{\prime}\right) \frac{1}{2} \rho \omega^{2} 2 \pi r^{2} d r \\
d T=4 a^{\prime}(1-a) \frac{1}{2} \rho V_{1}^{2} \omega r^{2} 2 \pi \mathrm{rdr}
\end{gathered}
$$

The thrust on an annular cross section can be also determined by the following expression that uses the axial induction factor, $a$ : 


$$
d I=4 a(1-a) \frac{1}{2} \rho V_{1}^{2} 2 \pi \mathrm{rdr}
$$

In order to obtain a relationship between the axial induction factor and the angular induction factor, Eqs. (21) and (23) can be equated, giving Eq. (24):

$$
\frac{a(1-a)}{a^{\prime}\left(1+a^{\prime}\right)}=\lambda_{r}^{2}
$$

where the local tip-speed ratio $\left(\lambda_{r}\right)$ is defined as Eq. (25):

$$
\lambda_{r}=\frac{\omega r}{V_{1}}=\lambda \frac{r}{R}
$$

On the other hand, the blade element theory is useful to derive expressions of developed torque and the axial thrust force experienced by the turbine. This theory is based on the analysis of the hydrodynamic force applied to a radial blade element of infinitesimal length. Figure 3 illustrates a transversal cut of the blade element. In the figure, the hydrodynamic force acting on the blade element is also depicted. The blade element moves in the water flow at a relative speed $\left(V_{\text {Rel }}\right)$. The flow around the blade starts at station 2 in Figure 2 and ends at station 3 . At the inlet to the blade, the flow is not rotating, and at the exit from the blade, the flow rotates at a rotational speed $(\Omega$.). That is, over the blade row, wake rotation has been introduced. The average rotational flow over the blade due to wake rotation is, therefore, $\Omega / 2$. The blade is rotating with speed $\omega$. The average tangential velocity that the blade experiences is, thus, $\omega r+\frac{1}{2} \Omega r$. This is shown in Figure 3 .

A blade can be divided into $\mathrm{N}$ elements as shown in Figure 3. Each of the blade elements will experience a slightly different flow as they have a different rotational speed $(\omega r)$ and a different chord length $(c)$. The water flow establishes a differential pressure around the blade element, which results in a force perpendicular to the local water movement direction, the so-called lift force $(\mathrm{dL})$. Additionally, a drag force $(\mathrm{dD})$ is done in the flow direction. The drag force is due to both viscous friction forces at the surface of the hydrofoil and the unequal pressure on the hydrofoil surface facing toward and away from the oncoming flow [9]. $d L$ and $d D$ can be found from the definition of the lift $\left(C_{L}\right)$ and drag $\left(C_{D}\right)$ coefficients as follows:

$$
\begin{aligned}
& \mathrm{dL}=C_{L} \frac{1}{2} \rho V_{R e l}{ }^{2} c d r \\
& d D=C_{D} \frac{1}{2} \rho V_{R e l}{ }^{2} c d r
\end{aligned}
$$

Both lift and drag coefficients are functions of the type of hydrofoil used for the blade and the incidence angle $(\alpha)$, defined as the angle that the flow makes with the chord. As observed in Figure 3, it is possible to obtain the distribution of the angle of the relative water velocity $(\phi)$ which consists of the pitch angle $(\theta)$, twist angle $(\beta)$, and angle of attack $(\alpha)$ on one of the blade sections (hydrofoil) by employing blade element momentum theory, as expressed in Eq. (28):

$$
\phi=\alpha+\theta+\beta
$$




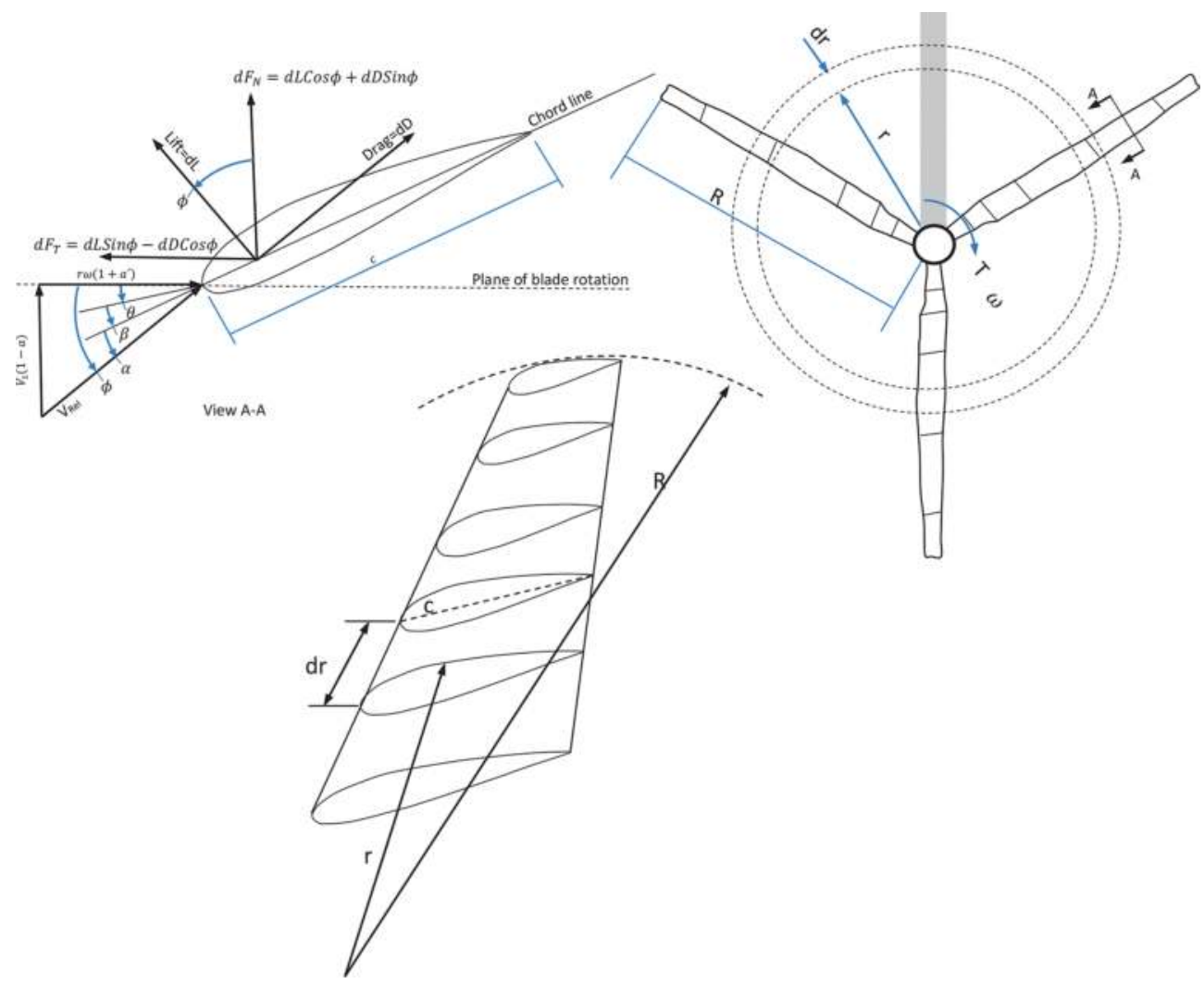

Figure 3. The blade element model.

where $\phi$ is the angle between the local flow direction and the rotor plane, and $\beta$ is the twist angle. However, $C_{p}$ has a maximum when $\theta$ is equal to 0 ; therefore, Eq. (28) can be rewritten as Eq. (29):

$$
\phi=\alpha+\beta
$$

\section{Examining Figure 3:}

$$
\operatorname{Tan} \phi=\frac{V_{1}(1-a)}{\omega r\left(1+a^{\prime}\right)}
$$

Substituting Eq. (25) into Eq. (30), the expression for Tan $\phi$ can be further simplified to Eq. (31):

$$
\operatorname{Tan} \phi=\frac{(1-a)}{\lambda_{r}\left(1+a^{\prime}\right)}
$$


From Figure 3, the relative water velocity can be expressed as a function of the water velocity and the rotational speed as expressed by Eqs. (32) and (33), respectively:

$$
\begin{gathered}
V_{R e l}=\frac{V_{1}(1-a)}{\operatorname{Sin} \phi} \\
V_{R e l}=\frac{\omega r\left(1+a^{\prime}\right)}{\operatorname{Cos} \phi}
\end{gathered}
$$

The lift and drag forces can be resolved into a normal force $\left(d F_{N}\right)$ to the plane of rotation (this contributes to thrust) and a tangential force $\left(d F_{T}\right)$ to the circle swept by the rotor. This is the force creating useful torque. Therefore, if there are $B$ number of blades, the torque $(d T)$ on the rotor of a hydrokinetic turbine is simply the tangential force multiplied by the radius:

$$
\begin{gathered}
d F_{N}=\mathrm{dLC} \operatorname{Cos} \phi+\mathrm{dD} \operatorname{Sin} \phi=\left(C_{L} \operatorname{Cos} \phi+C_{D} \operatorname{Sin} \phi\right) \frac{1}{2} \rho V_{R e l}{ }^{2} c d r \\
d F_{T}=\mathrm{dLSin} \phi-\mathrm{dD} \operatorname{Cos} \phi=\left(C_{L} \operatorname{Sin} \phi-C_{D} \operatorname{Cos} \phi\right) \frac{1}{2} \rho V_{R e l}{ }^{2} c d r \\
d I=B \frac{1}{2} \rho V_{R e l}{ }^{2}\left(C_{L} \operatorname{Cos} \phi+C_{D} \operatorname{Sin} \phi\right) c d r \\
d T=B \frac{1}{2} \rho V_{R e l}{ }^{2}\left(C_{L} \operatorname{Sin} \phi-C_{D} \operatorname{Cos} \phi\right) \mathrm{crdr}
\end{gathered}
$$

The power developed by the rotor $(d P)$ can be found as the product of the torque and the angular velocity, as expressed in Eq. (38):

$$
d P=B \omega \frac{1}{2} \rho V_{R e l}^{2}\left(C_{L} \operatorname{Sin} \phi-C_{D} \operatorname{Cos} \phi\right) \mathrm{crdr}
$$

In order to obtain the chord length (c), Eqs. (37) and (22) can be equated, resulting in Eq. (39):

$$
c=\frac{8 a^{\prime} \omega r^{2} \pi \operatorname{Sin}^{2} \phi}{\left(C_{L} \operatorname{Sin} \phi-C_{D} \operatorname{Cos} \phi\right) B(1-a)}
$$

The number of blades $(B)$ will affect the power output from the turbine. The optimum number of blades for a turbine depends on the purpose of the turbine. Turbines for generating electricity need to operate at high speeds, but they do not require much torque. These turbines generally have two or three blades, since this gives enough torque without adding the extra weight that can slow the turbine down. Rotors with odd numbers of blades, and at least three, are more stable. The advantage of having one or two blades in the rotor is the possible savings in production costs and weight. Nevertheless, the use of few blades requires a higher rotational speed or a larger chord length to yield the same energy output. A three-blade design also decreases the fluctuating loads from inertia variation [4, 5, 13-15].

In order to apply Eq. (39), it is necessary to introduce a corrective factor ( $\mathrm{Fc}$ ) for improving the structural strength of the blade. Fc was equal to 3.8. Thus, the values of each chord length calculated by Eq. (39) were multiplied by this factor. 
On the other hand, in the design of a blade, the most essential aspect is to choose a good profile. A blade profile is typically similar to the wing of an aircraft. The coordinates of hydrofoils are normally given as a function of the chord length $(x / c, y / c)$. These coordinates represent the profile, shape, and thickness of a blade at a particular section along the blade. In the blade design, an S822 hydrofoil profile was used $[13,14]$. This hydrofoil profile was developed under a joint effort between the National Renewable Energy Laboratory and Airfoils, Inc. It was specially tailored for using on small horizontal axis turbines. The hydrofoil has several advantages over those hydrofoils traditionally used on aircraft. First, the hydrofoils have a reduced roughness sensitivity for improving the energy capture under dirty blade conditions, owing to the accumulation of insect debris. Second, the increased section thickness of the root (S822, 16\%) and tip hydrofoil allows for a lower blade weight, lower cost, increased stiffness, and improved fatigue resistance.

Before using Eq. (39), $B, C_{L^{\prime}} C_{D^{\prime}} a, a^{\prime}$, and $\phi$ must be determined. In this work, $B$ was equal to three. The reasons for that selection were mainly the higher efficiency and smoother output torque than two-bladed turbines. In general, three blades are used for the turbine system to keep the dynamic balance and minimize the fatigue effect. The lift and drag coefficients can be obtained from wind tunnel test data for the type of the used airfoil. The values of $C_{L}(0.8)$ and $C_{D}(0.009)$ were used $[1,13,14] . \alpha$, a variable in Eq. (29), may also be found from test data, which in this case was $5^{\circ}$. The value of $a$ was determined from Eq. (18) for a $C_{p}$ and $\eta$ equal to 0.4382 and $70 \%$, respectively. In this case, $a$ was 0.15254 and $\phi$ can be worked out using Eq. (31). However, $\lambda_{r}$ must be first determined using Eq. (25).

Once $B, C_{L^{\prime}} C_{D^{\prime}} a, a^{\prime}$, and $\phi$ have been found, $c$ and $\beta$ can be calculated for any value of $r$ along the blade. In order to simplify, the whole blade length $(0.79 \mathrm{~m})$ was divided into 10 equal segments, separating each section by a distance of $0.079 \mathrm{~m}$. The sections were represented from $r_{1}$ to $r_{10}$, where $r_{1}$ was the section nearest to the root and $r_{10}$ was the tip of the blade. An Excel spreadsheet shown in Table 1 was used to obtain the values of $c$ and $\beta$ for each section. It is highlighted that the value of $c$ increases from the tip toward the root. $\beta$ was larger near the root and smaller at the tip, where local speeds are lower and higher, respectively. The conditions of the design produced an angular velocity equal to $114.68 \mathrm{rpm}$.

Once the chord length distribution and the twist distribution along the blade length have been found for a certain tip-speed ratio at which the power coefficient of the rotor is maximum, the next step is to multiply the value of $c$ at every section by the non-dimensional coordinates of the profile S822. The values of $x$ and $y$ coordinates of the profile for each section

\begin{tabular}{|c|c|c|c|c|c|c|c|}
\hline \multicolumn{8}{|c|}{ Predefined design parameters } \\
\hline $\begin{array}{l}\text { Turbine } \\
\text { output } \\
\text { power, } \\
P(w)\end{array}$ & $\begin{array}{l}\text { Fluid } \\
\text { density, } \rho \text {, } \\
\left(\mathrm{kg} / \mathrm{m}^{3}\right)\end{array}$ & $\begin{array}{l}\text { Water } \\
\text { velocity } V_{1^{\prime}} \\
(\mathrm{m} / \mathrm{s})\end{array}$ & $\begin{array}{l}\text { Power coefficient } \\
C_{p}\end{array}$ & $\begin{array}{l}\text { Efficiency } \\
\eta\end{array}$ & $\begin{array}{l}\text { Blade number } \\
B\end{array}$ & $\begin{array}{l}\text { Lift } \\
\text { coefficient, } \\
C_{L}\end{array}$ & $\begin{array}{l}\text { Drag } \\
\text { coefficient, } C_{D}\end{array}$ \\
\hline 1000 & 997 & 1.5 & 0.4382 & 0.7 & 3 & 0.8 & 0.009 \\
\hline $\begin{array}{l}\text { Tip-speed } \\
\text { ratio, } \lambda\end{array}$ & $\begin{array}{l}\text { Angle of } \\
\text { attack, } \alpha\end{array}$ & \multicolumn{3}{|c|}{ Axial induction factor, $a$ Eq. (18) } & $\begin{array}{l}\text { Rotational } \\
\text { speed, } \omega \\
(\mathrm{rad} / \mathrm{s}) \text { Eq. (6) }\end{array}$ & $\begin{array}{l}\text { Rotor radius, } \\
R(\mathrm{~m}) \text { Eq. }(5)\end{array}$ & $\Delta r$ \\
\hline 6.325 & $5^{\circ}$ & 1.291 & 0.153 & 0.556 & 12.099 & 0.79 & 0.079 \\
\hline
\end{tabular}




\begin{tabular}{llllllll}
\hline \multicolumn{7}{c}{ Computed parameters } \\
\hline $\begin{array}{l}\text { Station no. } \\
\begin{array}{l}1 \text { root, } \\
10 \text { tip) }\end{array}\end{array}$ & $\begin{array}{l}\text { Blade } \\
\text { element, } \\
\text { r (m) }\end{array}$ & $\begin{array}{l}\text { Local tip- } \\
\text { speed ratio, } \\
\text { Ar Eq. (25) }\end{array}$ & $\begin{array}{l}\text { Angular } \\
\text { a' Eq. }(24)\end{array}$ & $\begin{array}{l}\text { Relative } \\
\text { velocity } \\
\text { angle, } \phi \\
\text { (rad). Eq. } \\
(31)\end{array}$ & $\begin{array}{l}\text { Relative } \\
\text { velocity angle, } \\
\phi \text { (deg) }\end{array}$ & $\begin{array}{l}\text { Blade twist, } \\
\beta \text {. Eq. (29) }\end{array}$ & $\begin{array}{l}\text { Chord length, } \\
c \text { (m). Eq. (39) }\end{array}$ \\
\hline 1 & 0.079 & 0.633 & 0.257 & 0.817 & 46.83 & 41.83 & 0.117 \\
2 & 0.158 & 1.265 & 0.075 & 0.557 & 31.93 & 26.93 & 0.100 \\
3 & 0.237 & 1.898 & 0.035 & 0.407 & 23.35 & 18.35 & 0.078 \\
4 & 0.316 & 2.530 & 0.020 & 0.317 & 18.18 & 13.18 & 0.063 \\
5 & 0.395 & 3.163 & 0.013 & 0.259 & 14.82 & 9.82 & 0.053 \\
6 & 0.474 & 3.795 & 0.009 & 0.218 & 12.48 & 7.48 & 0.045 \\
7 & 0.553 & 4.428 & 0.007 & 0.188 & 10.77 & 5.77 & 0.039 \\
8 & 0.632 & 5.060 & 0.005 & 0.165 & 9.46 & 4.46 & 0.035 \\
9 & 0.711 & 5.693 & 0.004 & 0.147 & 8.43 & 3.43 & 0.032 \\
10 & 0.790 & 6.325 & 0.003 & 0.133 & 7.61 & 2.61 & 0.029 \\
\hline
\end{tabular}

Table 1. Excel spreadsheet for determining the section chord length and twist angle.

were exported to parametric 3D design software by using SolidWorks [13, 14]. Therefore, the cross sections of the blade were created at different distances from the root to the tip, and using the Loft command, a 3D model of the whole blade was produced. The resulting image is shown in Figure 4.

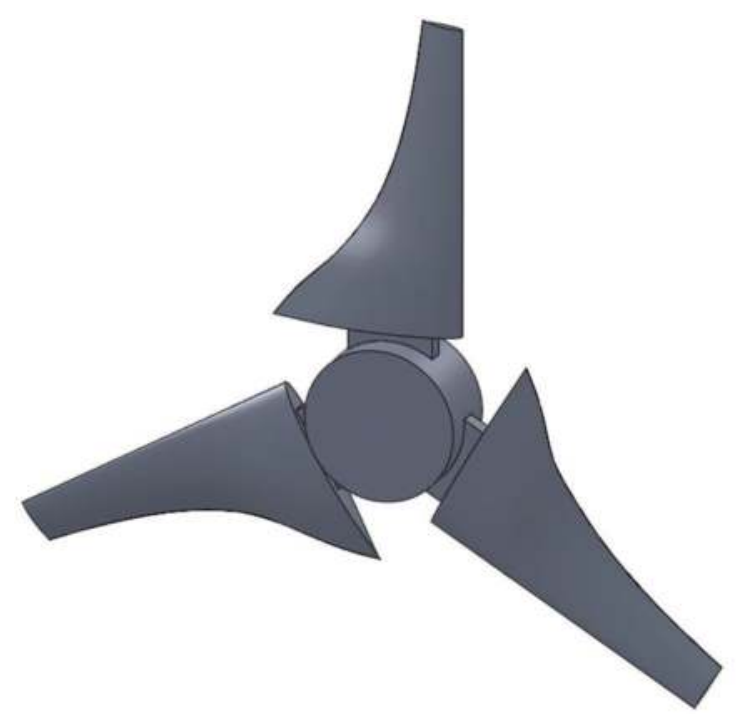

Power

Figure 4. Specifications of the turbine used for simulation studies. 


\section{Structural design of a horizontal axis hydrokinetic turbine}

The rotor hydrodynamic design was validated with a detailed three-dimensional CFD, performed in ANSYs Fluent, and FEA, performed in ANSYs Workbench. The fluid domain was coupled with the structural domain through one-way coupling, and a fluid-structure interaction analysis was carried out to find the effect of the blade geometry and the operating conditions on the stresses developed in the blades.

Based on the characteristic of cyclic symmetry for the structure of the hydrokinetic turbine rotor, a rotor sector conformed by a blade and an angle of $120^{\circ}$ of the hub was used to run the simulation. Accordingly, a rotationally periodic boundary condition was applied across the back and bottom surface of the computational domain shown in Figure 5, which was created using SolidWorks and meshed in ANSYs. The used mesh was an unstructured mesh with very fine prisms layer near the turbine wall. All dimensions of the boundary were given in terms of radius of the turbine. Since the studied turbine possesses $120^{\circ}$ periodicity, only one blade was modeled. Figure 2 shows the location of the turbine within the computational domain. The turbine rotational plane was located $2 \mathrm{R}$ away from the inlet, and the fluid domain extended $5 R$ behind the turbine rotational plane to capture the near and far wake effects. The study assumed steady and incompressible flow. The velocity inlet boundary condition was applied on the left surface of the domain with uniform axial (free stream) velocity of $1.5 \mathrm{~m} / \mathrm{s}$. A pressure outlet boundary condition was provided on the right surface with zero gauge pressure. Realizable k-e turbulence model was chosen. The turbulence intensity of $5 \%$ was applied. The

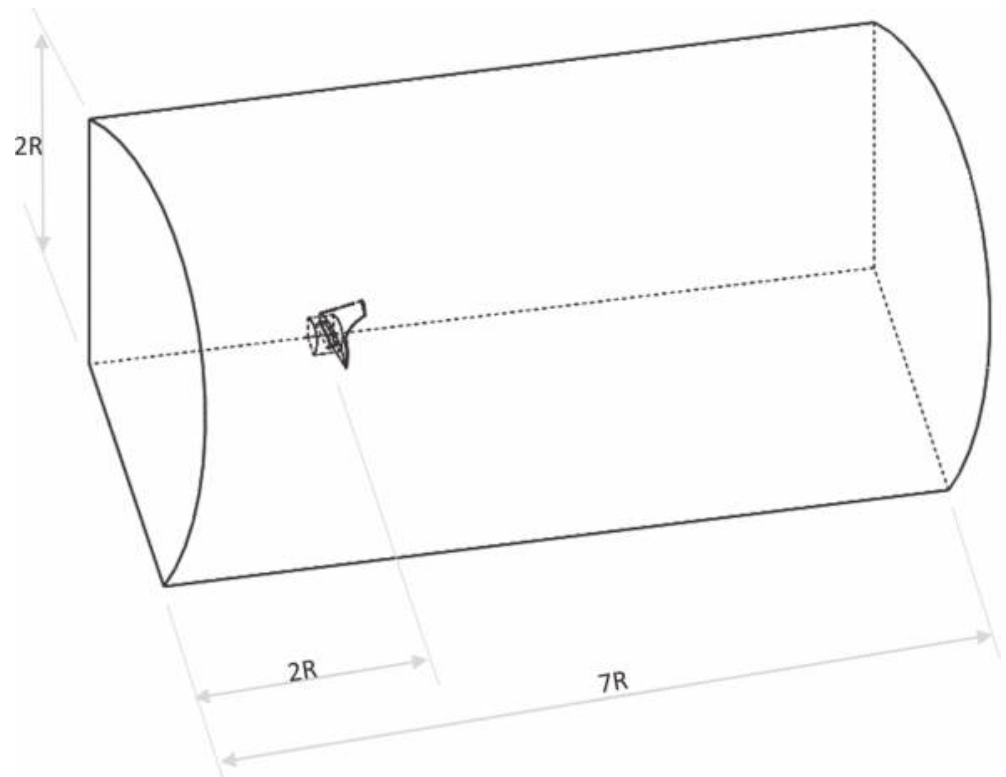

Figure 5. Computational domain used for the CFD analysis. 
no-slip condition was applied on the turbine blade and hub, i.e., the relative velocity of the surface blade and hub was set to zero.

A grid independence study was carried out to study the effect of the number of elements on the CFD analysis. The CFD solutions were deemed to have converged when the scaled residuals for all the solution values had dropped by at least six orders of magnitude. The moment on blade was monitored to ensure convergence of the solutions. The CFD simulations provided the flow field within the computational domain and also the blade pressure distribution, shown in Figure 6. It can be observed that the pressure on the lower side is relatively higher than the pressure on the upper side, as expected. This creates a net upward force, which produces a lift. Spatial integration of the pressure distribution yielded extreme load distributions, specifically the shearing forces and the bending moments, over the blade.

The simulation allowed computing the torque on the blade $(29.366 \mathrm{Nm})$, which was subsequently multiplied by the blade total number (3) and the angular velocity (12.009 rad/s). Thus, the turbine power output was calculated $(1057.969 \mathrm{~W})$. This value was compared to the design power $(1000 \mathrm{~W})$. The good agreement between the numerical and theoretical turbine power output enabled to validate the design of the rotor.

The blades of a hydrokinetic turbine must be analyzed for hydrodynamic, centrifugal, and gravitational loads experienced during their operating life. The hydrodynamic loads, i.e., the extreme flap and edgewise loads that the blade would likely encounter over its lifetime, were associated with extreme flow conditions and were determined using CFD software. The centrifugal load was located at the rotation center of the rotor (rotation velocity of $12.009 \mathrm{rad} / \mathrm{s}$ ), and the gravity was defined in the Static Structural module of ANSYs.

Fluid-structure interaction system was created to perform static analysis by using pressure loads on the blade from Fluent software, transferred them by mapping algorithm, and projected on blade surface geometry in the Static Structural module, following the global

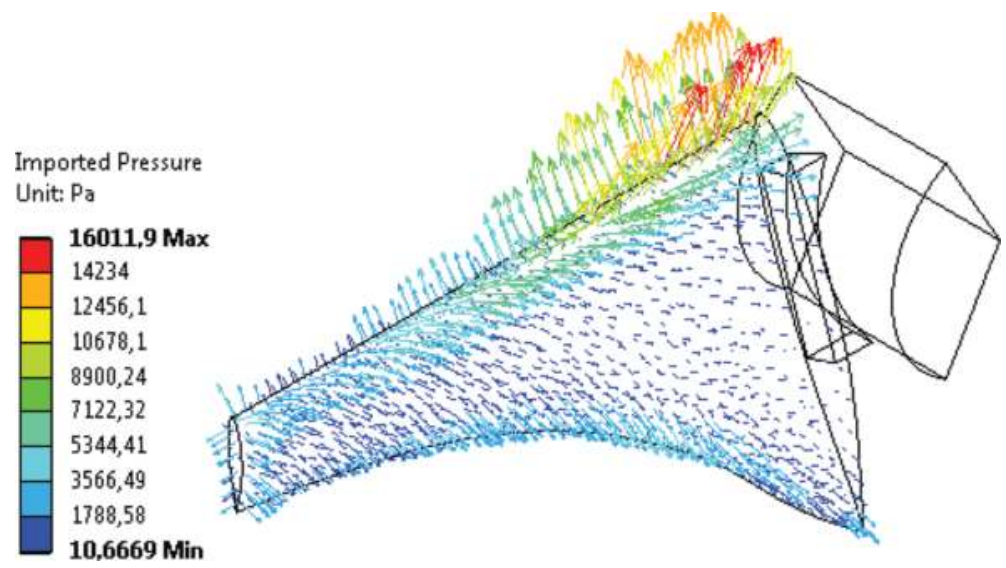

Figure 6. Blade pressure distributions for the extreme operating conditions. 
coordinate system (Figure 4). That is, the position of the blade geometry in Fluent module and Static Structural module must be the same one with reference to the global coordinate system. Figure 6 shows the "solution," e.g., the pressure loads on the blade, obtained from the Fluent module, which are connected to the "Setup" of Static Structural module and used as mechanical loads for the blade static analysis (Figure 7).

ANSYs Composite PrepPost (ACP) was used for the analysis of layered composite structures of the blade. ACP has a pre- and post-processing mode. In the pre-processing mode, all composite definitions can be created and mapped to the geometry (FE mesh). These composite definitions are transferred to the FE model (Static Structural module) and the solver input file. In the post-processing mode, after the solution is completed and the result file(s) are imported, postprocessing results (failure, safety, strains, and stresses) can be evaluated and visualized.

Various blade structural configurations, such as solid blade (Geometry A, Figure 8A), face sheet with core (Geometry B, Figure 8 B), and face sheet with shear webs and stations (Geometry C, Figure 8C), were investigated in order to know the global behavior of the blade in terms of its geometry, tip deflection, and stress levels.

The geometry B consisted of two faces or skins (suction and pressure sides) which provided the pressure distribution required for the blade rotation, and thus its cross section was in the form of specific hydrofoils due to hydrodynamic considerations associated with efficiency of the blade in capturing water energy. In addition to the two faces, it had a core made of

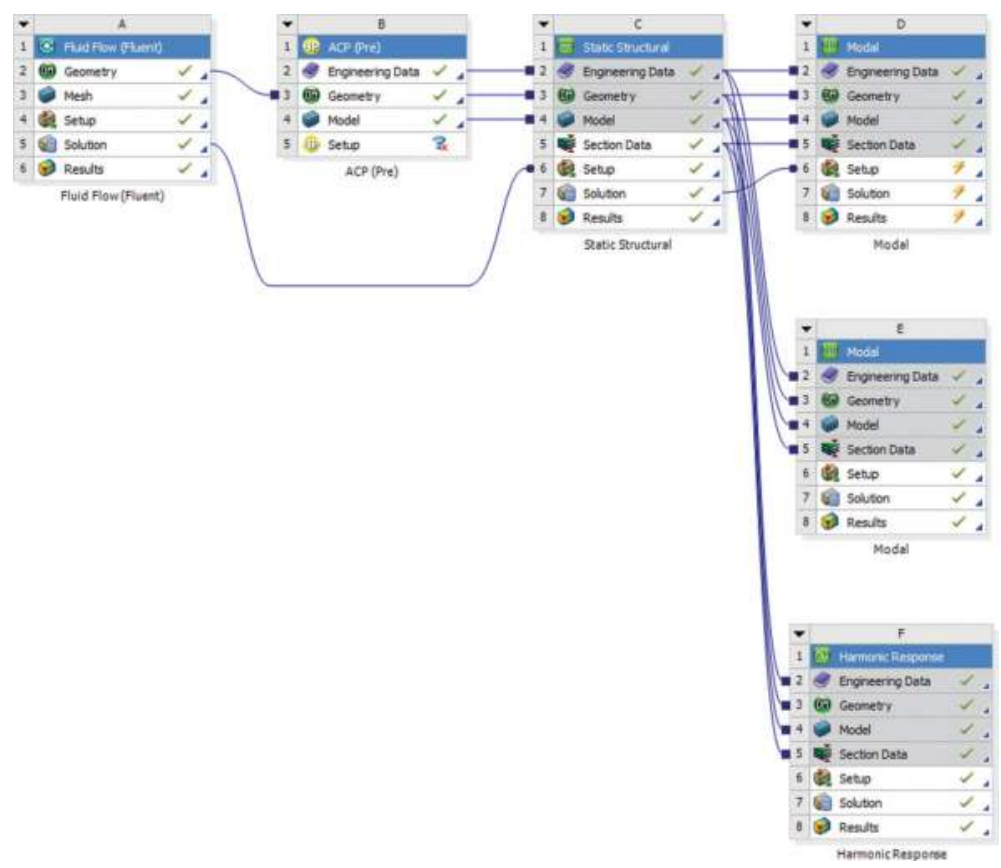

Figure 7. Schematic project of fluid-structure integration (FSI) in ANSYs Workbench. 


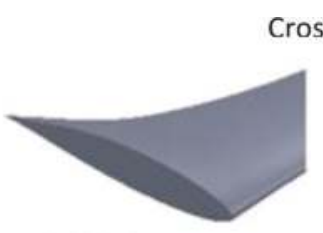

A) Solid blade

B) Facesheet with core
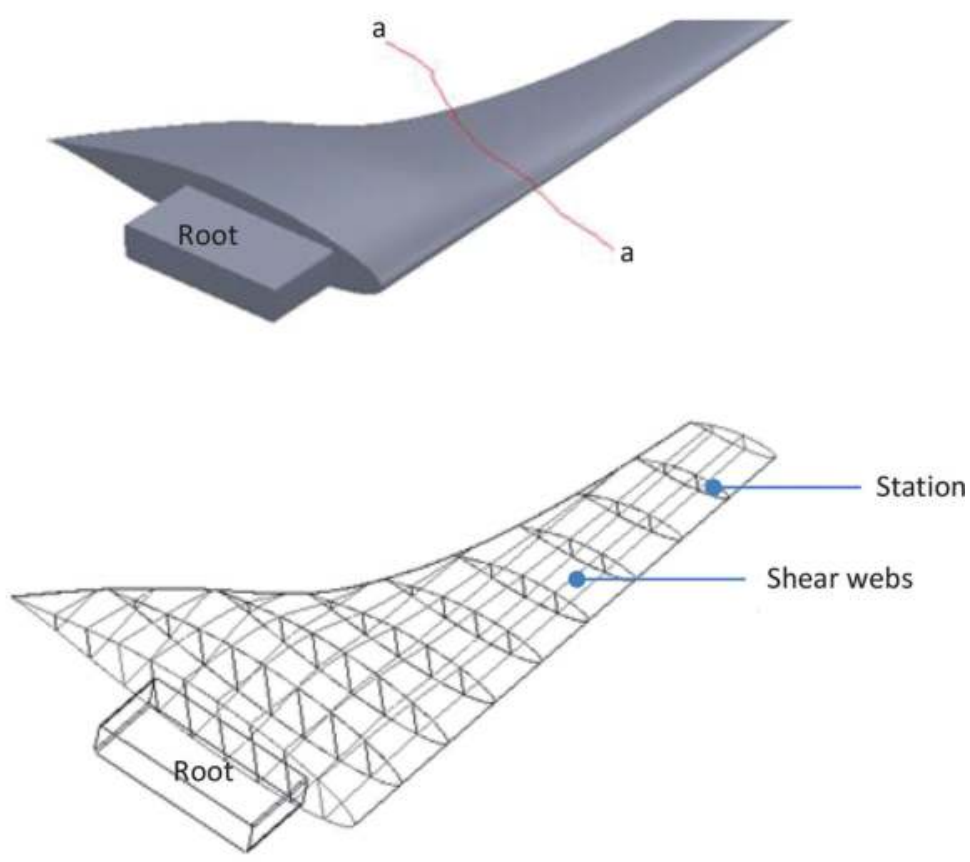

C) Skins (suction and pressure side) and several structural shear webs and stations

Figure 8. Structural models of the blade. (a) Geometry A, (b) Geometry B, and (c) Geometry C.

a material of low density. This core considerably contributed to the hydrodynamic performance of the blade. The geometry $\mathrm{C}$ consisted of two faces or skins (suction and pressure sides), structural shear webs, and several stations. The position, amount, and shape of the shear webs were important in the blade behavior. The shear webs and stations were located inside the shell formed by the faces, which were mainly responsible to support different load cases. The stations or transversal spars had a thickness of $2 \mathrm{~mm}$ and were connected to the webs. Since the webs were sheets of $2 \mathrm{~mm}$ thickness, they were easy to adjust to the stations to the attack angle of each hydrodynamic profile. The geometrical configurations of the blade were also modeled in SolidWorks. The models presented in Figure 8 include the $0.79 \mathrm{~m}$ long hydrodynamic blade. As it can be observed, this segment starts at a hub, with a rectangular cross section of height $0.035 \mathrm{~m}$ and width $0.170 \mathrm{~m}$ connected to the first hydrodynamic profile. 
The blade model was opened within ACP to define the material types, fiber orientations, and the construction of the layers for each region of the blade (geometries B and C). Since the properties of the composite were different in all of the three perpendicular directions (orthotropic), the model was sectioned into 22 different parts (2 skins, 10 stations, 9 shear webs, 1 root or hub), each one with their own material coordinate system. The skins, shear webs, and stations were modeled as a double laminated ply with a total thickness of $2 \mathrm{~mm}$; each ply would be $1 \mathrm{~mm}$ thick with different orientations.

The orthotropic material property descriptions, material thicknesses, and fiber orientations were entered into a material data folder in ACP, where "materials," "fabrics," and "stackups" were stored. From the Workbench project page, the loads imported from the CFD analysis, the boundary conditions, and the specific analysis options were applied to the model, and the analysis was executed. Results may be post-processed in mechanical or in ACP to see the stress and strain results in detail on a layer-by-layer basis.

Static numerical models for each geometry of the blade were created: (a) a solid blade (Figure 8A) made of nylon; (b) a shell-type blade with skins and a core (Figure 8B), whose skins were made of glass fiber (type E), the root of aluminum 6061-T6, and the core of balsa wood; and (c) a shell-type blade (with skins, webs, and stations, see Figure $8 \mathrm{C}$ ), where the skins, the webs, and the stations were made of glass fiber (type E) and the root of aluminum 6061-T6. The mechanical properties of the materials used in the simulation are listed in Table $2[10,16]$.

The unstructured tetrahedral mesh of the solid blades for the geometry A converged with 12,896 elements and 15,990 nodes. On the other hand, the structure of the blade for the geometry B was meshed with a mixed mesh of tetrahedral elements (root and core) and hexahedral elements (skins). This mesh converged with 13,053 elements and 15,842 nodes. The geometry $\mathrm{C}$ was also meshed with a mixed mesh of tetrahedral elements (root) and hexahedral elements (skins, webs, and stations). This mesh converged with 10,308 elements and 10,419 nodes. The thickness of the skins, weds, and stations was $2 \mathrm{~mm}$.

Symmetrical and identical composite lay-up was assumed for skins, shear webs, and stations to improve convergence and achieve a balanced structural design. Two types of ply orientation $(0 / 90$ and \pm 45$)$ for the skins, webs, and stations were chosen for the geometries $\mathrm{B}$ and $\mathrm{C}$ based on practical selections of ply orientation in industry [16-18].

All the blade models were treated as a cantilevered beam with all the degrees of freedom fixed at the hub. Centrifugal, gravitational, and hydrodynamic forces were used for structural analysis. The von Mises stress and displacements were calculated for different models.

The results for maximum von Mises stresses and displacement over the complete geometry of the blade are presented in Table 3. None of the stresses shown in any region of the blade reached the limit values concerning the mechanical strength of the material for yielding. Stresses were maximum at the blade root for all the geometries.

Static structural analysis of geometries B and C shows that the ply orientation of fiber at \pm 45 was structurally superior in comparison with the ply orientation of fiber at $0 / 90$. The results 


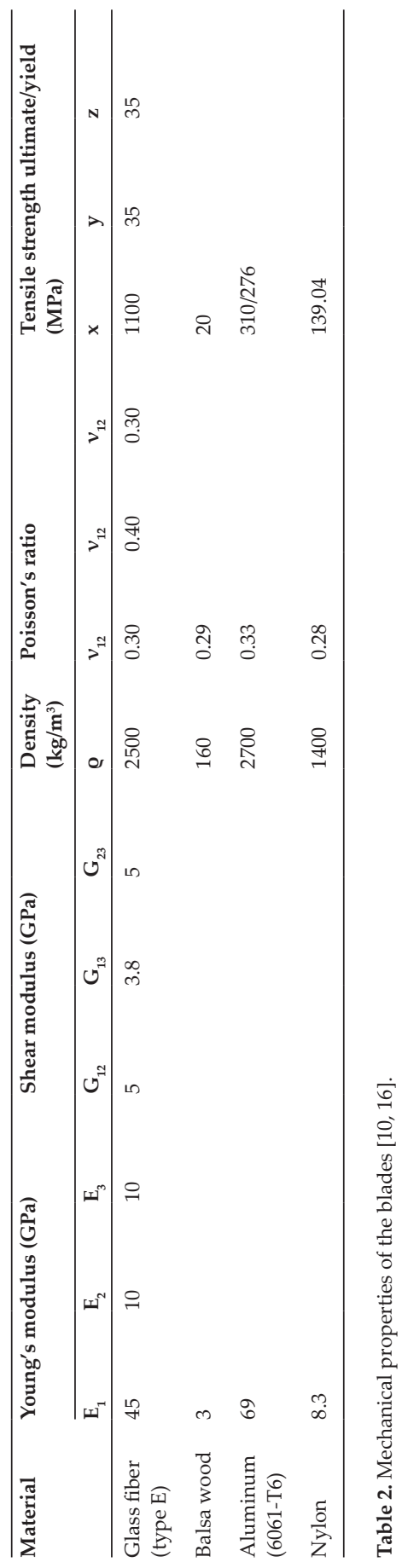




\begin{tabular}{|c|c|c|c|c|c|c|c|}
\hline \multirow{2}{*}{$\begin{array}{l}\text { Geometry } \\
\text { A }\end{array}$} & \multirow{2}{*}{$\begin{array}{l}\text { Part } \\
\text { All the blade }\end{array}$} & \multirow{2}{*}{$\begin{array}{l}\text { Material } \\
\text { Nylon }\end{array}$} & \multicolumn{2}{|c|}{$\begin{array}{l}\text { Von Mises stress } \\
\text { (Mpa) }\end{array}$} & \multicolumn{2}{|c|}{$\begin{array}{l}\text { Displacement } \\
\text { results }(\mathrm{mm})\end{array}$} & \multirow{2}{*}{$\begin{array}{l}\begin{array}{l}\text { Weight } \\
\text { (kg) }\end{array} \\
8.2628\end{array}$} \\
\hline & & & 2.3228 & & 2.1589 & & \\
\hline \multirow[t]{5}{*}{ B } & \multirow[t]{2}{*}{ Composite structures of the blade } & & \multicolumn{5}{|c|}{ Ply orientation } \\
\hline & & & {$[0 / 90]$} & {$[ \pm 45]$} & {$[0 / 90]$} & {$[ \pm 45]$} & \\
\hline & Skins (suction and pressure side) & Glass fiber (type E) & & & & & \\
\hline & Core & Balsa wood & 9.9773 & 9.0558 & 0.87592 & 1.2126 & 11.0470 \\
\hline & Root & Aluminum (6061-76) & & & & & \\
\hline \multirow[t]{3}{*}{ C } & Skins (suction and pressure side) & Glass fiber (type E) & & & & & \\
\hline & Shear webs and stations & Glass fiber (type E) & 43.1840 & 33.3000 & 1.7754 & 2.4785 & 11.3010 \\
\hline & Root & Aluminum (6061-76) & & & & & \\
\hline
\end{tabular}

Table 3. Structural simulation results of the blade.

summarized in Table 2 show that the blade made up of glass fiber did not fail structurally, due to the stresses produced in all the parts of the analyzed models with glass fiber composite material did not exceed the safe working stresses of this material. Additionally, after the structural design of geometry B it was confirmed that the failure in the core of balsa wood did not occurred through von Mises' criterion. Figure $\mathbf{9}$ shows the variation of von Mises stress, displacement, and weight with respect to the analyzed geometries.

On the other hand, the main cause in hydrokinetic turbine blade failure is the high fatigue cycle. Fatigue failure is related to repeated cycling of the load on a structural member. Generally, the fatigue life of a structural member, i.e., the number of load cycles it can survive, is determined by the magnitude of the stress cycles. The exact relation between the magnitude of the stress and the fatigue life depends on the material properties of the structural member. In general, higher stresses lead to a shorter fatigue life. For some materials, fatigue only occurs if stresses exceed a minimum level, while for other materials there is no minimal stress level [4, 19-23].

Fatigue failure occurs when a crack is initiated, which results after a number of load cycles, leading to the concentration of high stress at a specific point of the material, known as point with relatively rough or soft surfaces or with sharp geometrical discontinuities. This crack advances slowly through the material surface up to a certain point from which it is accelerated until the final failure occurs. It is important to note that a high-cycle fatigue corresponds to failure after a relatively large number of load cycles, resulting in stress levels well below the generated material strength, exhibiting an elastic deformation. Furthermore, it is noteworthy that a structural member failure may not be attributed to the excess of load but to the repeated cycling loading $[4,5,19-23]$.

In principle, there are two ways in which the failure of turbine blades due to fatigue problems can be eliminated: (a) when an appropriate structural design is performed and (b) when the cycling of the load is presented. The correct design of a structural member can usually 


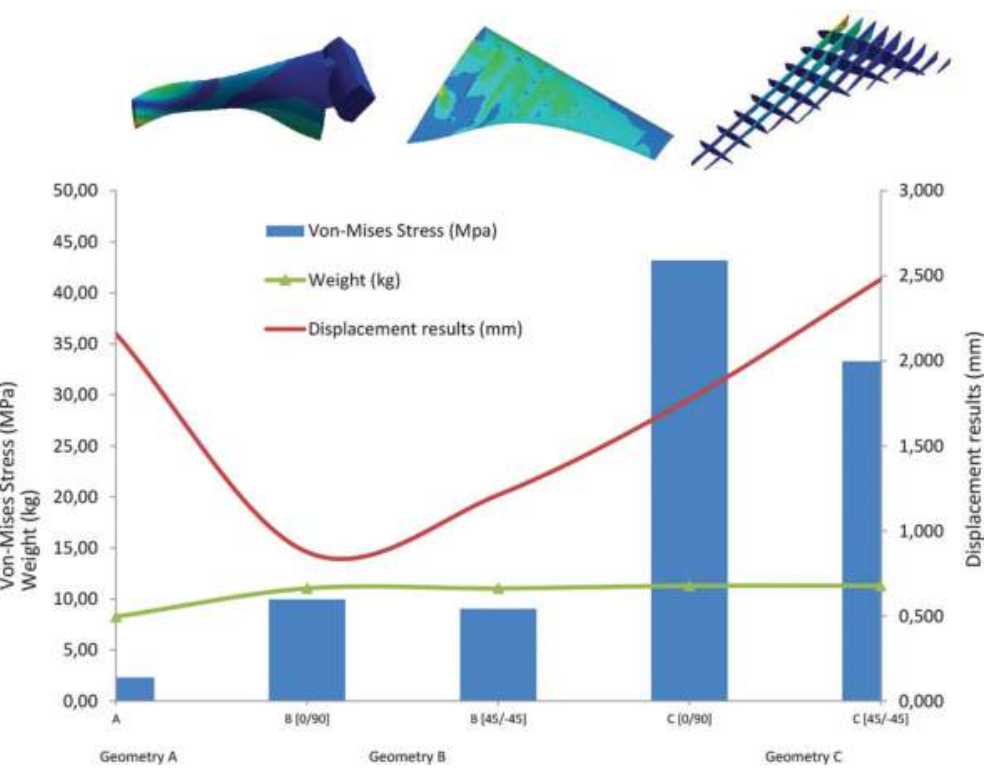

Figure 9. Von Mises stress and displacement on the blade.

eliminate or dramatically reduce fatigue problems. Nevertheless, for a turbine blade, this is not always possible. The design of a blade is usually constrained by hydrodynamic properties, weight, rotor length, etc., which can make the elimination of fatigue problems through design modifications very difficult if not impossible. On the other hand, the prevention of cyclic loading in hydrokinetic turbines is virtually impossible in practice $[19,20]$.

Modal analysis can be a powerful tool to assist in the identification and elimination of fatigue problems. The most obvious use of modal analysis is the determination of the natural frequencies of the turbine blades. The knowledge of these frequencies can be very useful in avoiding excessive excitations and thereby reducing the risk of fatigue failure. Computer-generated models of the turbine blades can be very useful to investigate the turbine and turbine blade properties under running conditions. Therefore, the modal analysis of the blade was performed using also ANSYs Workbench to extract the natural frequencies and the dominant modes using block Lanczos method [19-21].

For the modal analysis of the blade, loads and constraints were applied as in the structural analysis. First, six significant natural frequencies were calculated for both the cases; i.e., with press stress on and with press stress off. Pre-stressed analysis was performed by doing a static analysis before the modal analysis so that all the stresses and displacements, which are created by the centrifugal, hydrodynamic, and gravitational loads, are included into the modal analysis. In static analysis, rotational speed (in rad/s) was applied.

Results from modal analysis were not evidenced to be highly affected when loads were applied. The comparison of both the results is given in Table 4. It can be observed that both 


\begin{tabular}{|c|c|c|c|}
\hline Geometry & Mode no. & $\begin{array}{l}\text { Natural frequencies with } \\
\text { press stress effect }(\mathrm{Hz})\end{array}$ & $\begin{array}{l}\text { Natural frequencies with } \\
\text { press stress effect }(\mathrm{Hz})\end{array}$ \\
\hline \multirow[t]{6}{*}{ A } & 1 & 59.413 & 59.344 \\
\hline & 2 & 169.08 & 169.01 \\
\hline & 3 & 267.07 & 267.05 \\
\hline & 4 & 378.46 & 378.38 \\
\hline & 5 & 467.46 & 467.32 \\
\hline & 6 & 645.85 & 645.79 \\
\hline \multirow[t]{6}{*}{ B $( \pm 45)$} & & 98.874 & 98.828 \\
\hline & & 312.790 & 312.750 \\
\hline & & 399.340 & 399.320 \\
\hline & & 761.600 & 761.560 \\
\hline & & 817.630 & 817.540 \\
\hline & & 1105.400 & 1105.400 \\
\hline \multirow[t]{6}{*}{ B $(0 / 90)$} & & 117.200 & 117.160 \\
\hline & & 353.670 & 353.660 \\
\hline & & 441.970 & 441.950 \\
\hline & & 661.120 & 661.010 \\
\hline & & 889.220 & 889.160 \\
\hline & & 1055.500 & 1055.400 \\
\hline \multirow[t]{6}{*}{$C( \pm 45)$} & & 70.366 & 70.302 \\
\hline & & 199.530 & 199.490 \\
\hline & & 264.710 & 264.620 \\
\hline & & 345.760 & 345.700 \\
\hline & & 443.360 & 443.330 \\
\hline & & 489.900 & 489.870 \\
\hline \multirow[t]{6}{*}{$C(0 / 90)$} & & 83.066 & 83.010 \\
\hline & & 215.660 & 215.590 \\
\hline & & 299.160 & 299.100 \\
\hline & & 400.100 & 400.040 \\
\hline & & 462.320 & 462.150 \\
\hline & & 510.930 & 510.900 \\
\hline
\end{tabular}

Table 4. Modal analysis results. 
of the results are similar. The resulting mode shapes evidenced for the blades were flexural, axial, or edge bending and torsional modes. The rotational speed of the hydrokinetic turbine designed was $114.68 \mathrm{rpm}$, so the rotational frequency was $1.911 \mathrm{~Hz}$, resulting from $114.68 / 60$. There were three blades in total in the designed hydrokinetic turbine; therefore, the triple rotational frequency was $5.733 \mathrm{~Hz}$. This rotational frequency and triple rotational frequency are both far smaller than the first six frequencies of the five analyzed models. Thus, the designed hydrokinetic turbine rotors cannot be destroyed by resonance, which satisfies the design demand. As it is widely known, it must be noted that the phenomenon of resonance occurs if the natural frequency of structural system matches the frequency of dynamic loading, which may result in failure of structure. Mode shapes obtained through FEA are presented in Figure 10. These mode shapes were computed for a rotational speed of $1.911 \mathrm{~Hz}$. The mode shape contour plots are presented in the form of sum of deformations in the three orthogonal directions in Figure 10. It may be observed that the nature of these mode shapes remained largely unaffected by the rotational speed.

Changes in the blade natural frequencies with respect to the machine speed were plotted on a Campbell diagram (Figure 11). Critical speeds occur if the excitation frequencies coincide with the natural frequency of the rotating structure. The excitation frequency itself may be
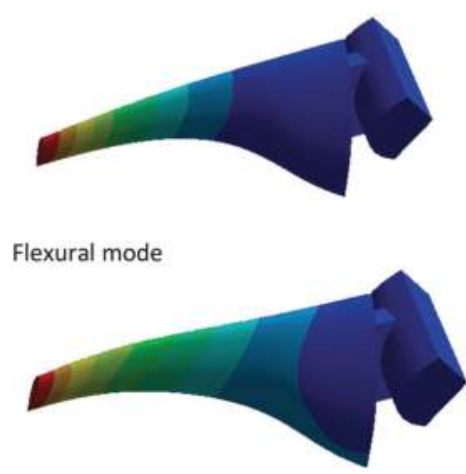

Flexural mode

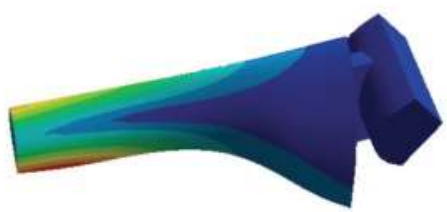

Torsional mode

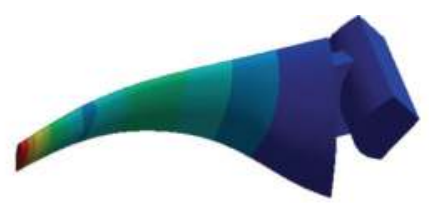

Flexural mode

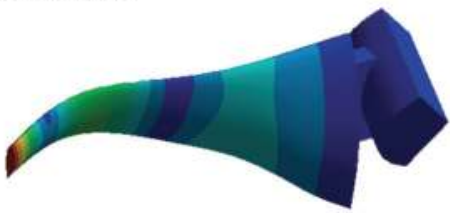

Flexural mode

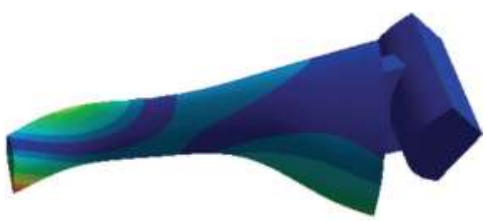

Torsional and edge bending mode

Figure 10. Mode shapes of the turbine blade. 


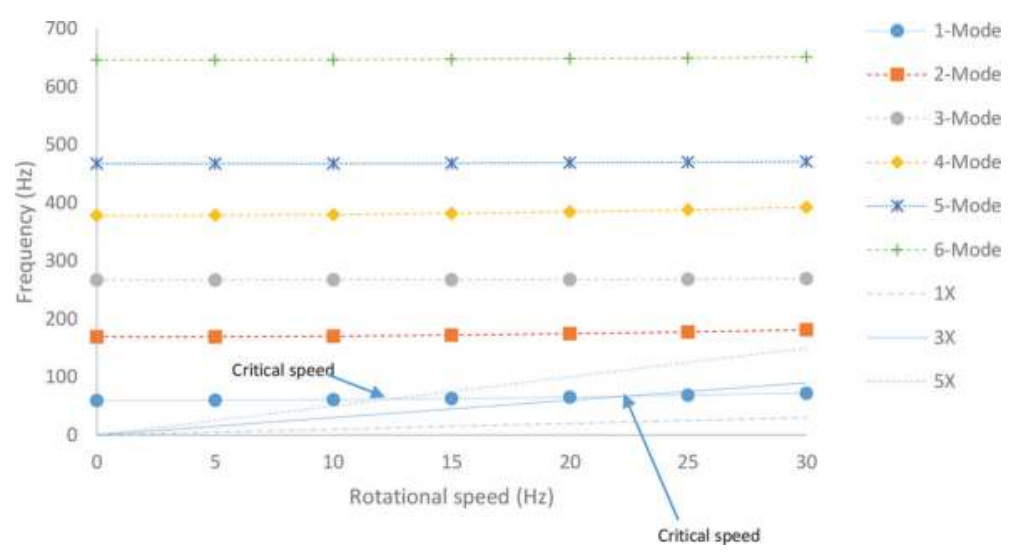

Figure 11. Campbell diagram of the turbine blade for geometry A.

synchronous to the rotational speed (1X) or multiples of it $(2 X, 3 X, 4 X, 5 X$, etc.). These excitation frequencies were represented as straight lines in the Campbell diagram. The intersections of these lines with the natural frequency curves indicated the critical speed. Using this procedure, critical speeds for different excitation frequencies were computed. The critical speeds were $22.407 \mathrm{~Hz}$ and $12.612 \mathrm{~Hz}$ for 3X and 5X the critical speed, respectively.

To finally calculate the dynamic response of the blade, the mode superposition harmonic analysis was applied. As it is widely known, it must be highlighted that harmonic response analysis is a technique used to determine the steady-state response of a linear structure to loads that vary sinusoidally, i.e., harmonically, with time [20-22]. Harmonic response analysis gives the ability to predict the sustained dynamic behavior of structures; enabling, subsequently, to verify whether or not the designs will successfully overcome resonance, fatigue, and other harmful effects of forced vibrations. Harmonic analysis uses the natural frequencies and mode shapes from the modal analysis. In this case, the frequency spectrum was set between 0 and $700 \mathrm{~Hz}$ with 100 steps for the geometry A.

It is highlighted that harmonic analyses require cyclic load data for the analysis. This load data were previously gathered from the hydrodynamic analyses of the turbine carried out by using CFD and static analysis. Therefore, the forces considered in this case were the blade selfweight and the maximum pressure due to the hydrodynamic load. A critical stress region was found from the stress contour obtained by performing harmonic analysis. The stress contours were plotted for the first and second natural frequencies $(59.413 \mathrm{~Hz}$ and $169.08 \mathrm{~Hz}$, respectively). The maximum stresses observed at these frequencies were $96.147 \mathrm{kPa}$ and $3.015 \mathrm{MPa}$, respectively. The region of critical stress resulted to be near the root for natural frequencies of 59.4313 and near the tip for frequencies of $169.08 \mathrm{~Hz}$. The von Mises stress variations with frequency at these critical nodes are plotted in Figure 12. As observed from these results, the stress in the material was well below the endurance limit. Thus, the structure may be considered safe for the presented loads.

Amplitude versus frequency was plotted using the post-processing option in ANSYs. The frequency response function (FRF) for this analysis is shown in Figure 13. These graphics are 


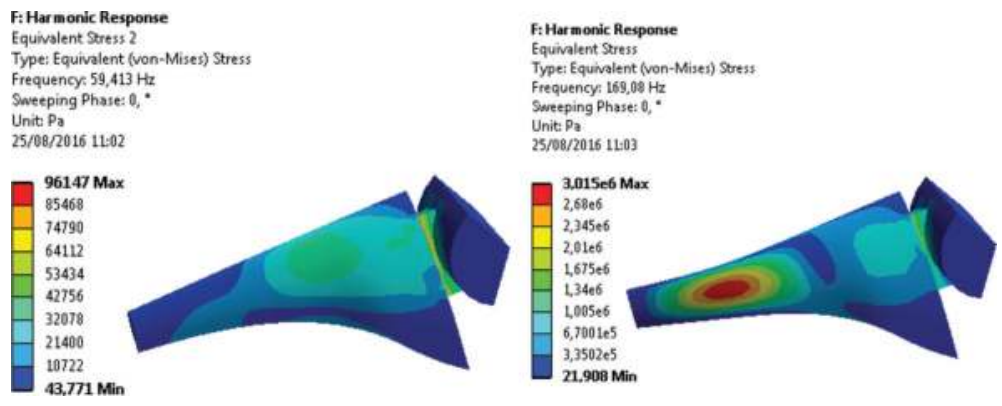

Figure 12. Stress contour (in $\mathrm{MPa}$ ) at the natural frequency corresponding to $1 \mathrm{~F}$ mode $(59.413 \mathrm{~Hz})$ and $2 \mathrm{~F}$ mode (169.08 Hz).
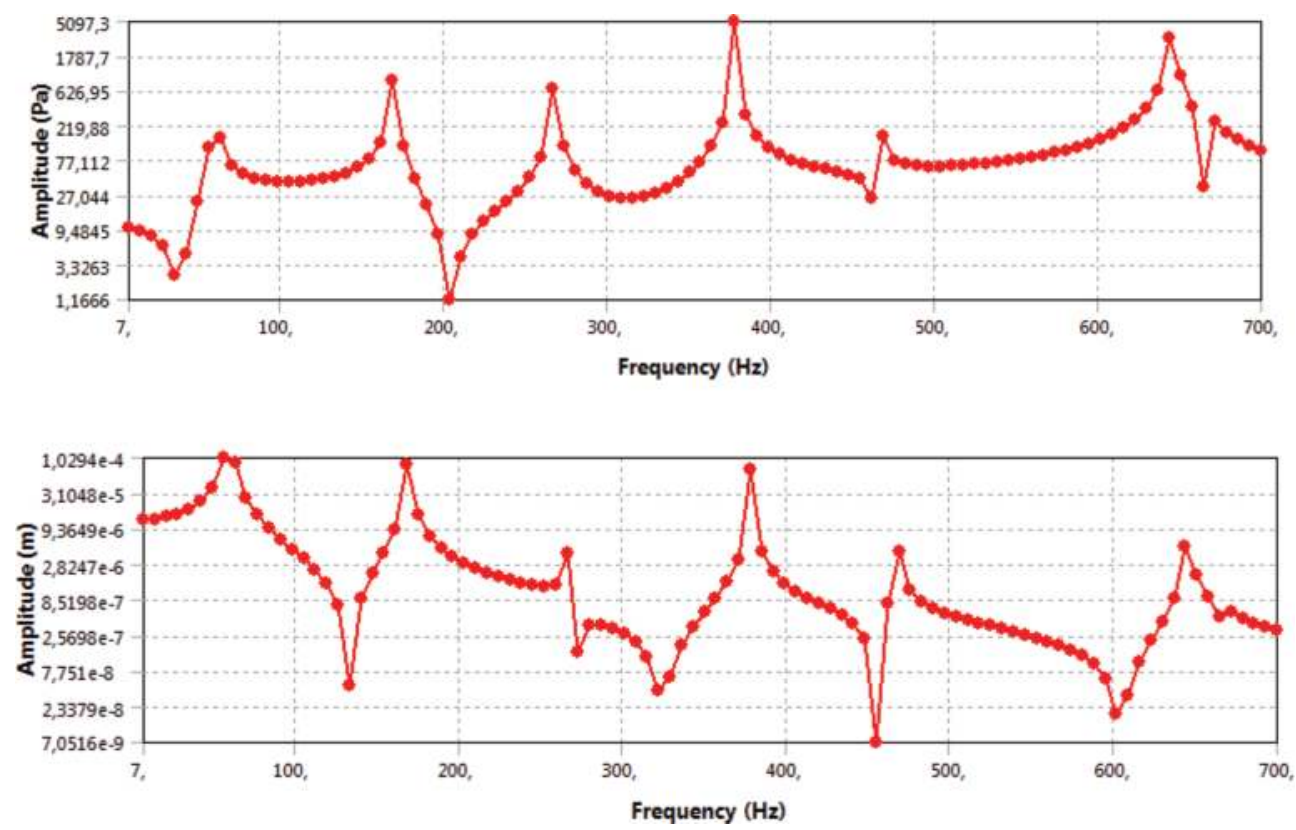

Figure 13. Variation of stress amplitude and displacement amplitude with different exciting frequencies.

specific for all the examined blades. The maximum displacement value indicated by FRF was very small as compared to the maximum displacement value calculated using static analyses of turbine (2.1589e-3 m), under which the turbine blade is safe for operation. In addition, FRF indicated no chances of the occurrence of resonance within this range.

\section{Conclusion}

The blades represent a vital component of any hydrokinetic turbine due to their complexity, cost, and significant effect on the operating efficiency. During its lifetime, a hydrokinetic turbine blade 
is subject to different types of loads, such as hydrodynamic, inertial, and gravitational forces. The hydrodynamic design provided the blade external shape, i.e., the chord and twist angle distributions along the blade, which resulted in optimal performance of the hydrokinetic turbine over its lifetime.

The structural design of the blades of a hydrokinetic turbine is paramount to ensure they are rugged, robust, and reliable and yet effective at capturing maximum available energy in the hostile environment of water currents. Therefore, a coupled fluid-structure interaction analysis for a horizontal axis hydrokinetic turbine of $1 \mathrm{~kW}$ was carried out using the software ANSYs Workbench to know the effect of several blade structure shapes and different materials on the stresses developed on blades due to the influence of the operating loads. ANSYs$\mathrm{ACP}$ was used as pre-processor for structural analysis of layered composite geometries B and C. FEA indicated that the turbine blade geometries might satisfactorily support the force exerted on it under the combined effects of the centrifugal, gravitational, and hydrodynamic studied loads, since for the analyzed external loads on the blade the stresses were observed to be still below the yield stress of the applied materials, preventing the blades from failure.

Additionally, the dynamic characteristics of the hydrokinetic turbine blade are important to ensure that the blade is rotating at frequencies as far as possible from its natural frequencies to prevent resonance phenomena. Mode shapes generated from modal analysis showed that different frequencies produced different characteristics, including torsional, bending, and combination of torsional and bending modes. From modal analysis and harmonic analysis, it can be observed that the first natural frequencies were far higher than the maximum operating frequencies, leading to a clear indication that the turbine is safe from resonance phenomenon. The harmonic responses within the specified range were also acceptable, as the maximum values of displacement were far lesser than the static displacement values of the analyzed models.

\section{Acknowledgements}

The authors gratefully acknowledge the financial support from the Colombian Institute of Science and Technology (COLCIENCIAS) and the Universidad de Antioquia.

\section{Author details}

Edwin Chica ${ }^{1 *}$ and Ainhoa Rubio-Clemente ${ }^{2}$

*Address all correspondence to: edwin.chica@udea.edu.co

1 Departamento de Ingeniería Mecánica, Facultad de Ingeniería, Universidad de Antioquia, Medellín, Colombia

2 Grupo de Diagnóstico y Control de la Contaminación (GDCON), Facultad de Ingeniería, Sede de Investigaciones Universitarias (SIU), Universidad de Antioquia UdeA, Medellín, Colombia 


\section{References}

[1] M. Anyi and B. Kirke. Evaluation of small axial ow hydrokinetic turbines for remote communities. Energy for Sustainable Development. 2010;14(2):110-116.

[2] R.H. van Els and A.C.P.B. Junior. The Brazilian experience with hydrokinetic turbines. Energy Procedia Clean, Efficient and Affordable Energy for a Sustainable Future: The 7th International Conference on Applied Energy (ICAE2015), Abu Dhabi, United Arab Emirates. 2015;75:259-264.

[3] E. Rosenberg, A. Lind, and K.A. Espegren. The impact of future energy demand on renewable energy production case of Norway. Energy. 2013;61:419-431.

[4] H.J. Vermaak, K. Kusakana, and S.P. Koko. Status of micro-hydrokinetic river technology in rural applications: a review of literature. Renewable and Sustainable Energy Reviews. 2014;29:625-633.

[5] M.S. Gney and K. Kaygusuz. Hydrokinetic energy conversion systems: a technology status review. Renewable and Sustainable Energy Reviews. 2010;14(9):2996-3004.

[6] M.J. Khan, G. Bhuyan, M.T. Iqbal, and J.E. Quaicoe. Hydrokinetic energy conversion systems and assessment of horizontal and vertical axis turbines for river and tidal applications: a technology status. Applied Energy. 2009;86(10):1823-1835.

[7] D. Kumar and S. Sarkar. A review on the technology, performance, design optimization, reliability, techno-economics and environmental impacts of hydrokinetic energy conversion systems. Renewable and Sustainable Energy Reviews. 2016;58:796-813.

[8] M. Anyi and B. Kirke. Hydrokinetic turbine blades: design and local construction techniques for remote communities. Energy for Sustainable Development. 2011;15(3):223-230.

[9] J.F. Manwell, J.G. McGowan, and A.L. Rogers. Wind Energy Explained: Theory, Design and Application. Wiley, Chichester. 2009.

[10] A.H. Munoz, L.E. Chiang, and E.A. De la Jara. A design tool and fabrication guidelines for small low cost horizontal axis hydrokinetic turbines. Energy for Sustainable Development. Wind Power Special Issue. 2014;22:21-33.

[11] J.G. Slootweg, H. Polinder, and W.L. Kling. Dynamic modelling of a wind turbinewith doubly fed induction generator. In Power Engineering Society Summer Meeting, Vancouver, Canada. 2001;1:644-649.

[12] G. Riegler. Principles of energy extraction from a free stream by means of wind turbines. Wind Engineering. 1983;7(2):115-126.

[13] E. Chica, F. Pérez, A. Rubio-Clemente, and S. Agudelo. Design of a Hydrokinetic Turbine. WIT Transactions on Ecology and the Environment. Wessex Institute of Technology, UK. 2015;195:137-148. 
[14] E. Chica, F. Pérez, and A. Rubio-Clemente. Rotor structural design of a hydrokinetic turbine. International Journal of Applied Engineering Research. 2016;11(4):2890-2897.

[15] J.N. Goundar and M.R. Ahmed. Numerical and experimental studies on hydrofoils for marine current turbines. Renewable Energy 2012;42:173-179.

[16] Matweb. Material Property Data. http://www.matweb.com. 2015.

[17] D.M. Grogan, S.B. Leen, C.R. Kennedy, and C.M. Brdaigh. Design of composite tidal turbine blades. Renewable Energy 2013;57:151-162.

[18] S.H. Pierson. Composite Rotor Design for a Hydrokinetic Turbine. University of Tennessee Honors Thesis Projects, University of Tennessee Knoxville, Tennessee, 2009.

[19] H. Li, Z. Hu, K. Chandrashekhara, X. Du, and R. Mishra. Reliability-based fatigue life investigation for a medium-scale composite hydrokinetic turbine blade. Ocean Engineering. 2014;89:230-242.

[20] S. Saad, A. Behzad, and M. Amir. Dynamic analysis of a $5 \mathrm{KW}$ wind turbine blade with experimental validation. Journal of Space Technology. 2014;4(1):82-87.

[21] N. Tenguria, N.D. Mittal, and S. Ahmed. Modal analysis for blade of horizontal axis wind turbine. Asian Journal of Scientific Research 2011; 4:326-334.

[22] A. Gangele and S. Ahmed. Modal analysis of S809 wind turbine blade considering different geometrical and material parameters. Journal of the Institution of Engineers (India): Series C. 2013;94(3):225-228.

[23] C. Liu, D. Jiang, and J. Chen. Vibration characteristics on a wind turbine rotor using modal and harmonic analysis of FEM. In 2010 World Non-Grid-Connected Wind Power and Energy Conference. IEEE, Nanjing, China. 2010:1-5. 